Article

\title{
Polysaccharide-Enriched Fraction from Amillariella Mellea Fruiting Body Improves Insulin Resistance
}

\author{
Siwen Yang ${ }^{\dagger}$, Yuhan Meng ${ }^{\dagger}$, Jingmin Yan, Na Wang, Zhujun Xue, Hang Zhang and \\ Yuying Fan * (D)
}

School of Life Sciences, Northeast Normal University, Jilin 130024, China; yangsw776@nenu.edu.cn (S.Y.); mengyh242@nenu.edu.cn (Y.M.); yanjm339@nenu.edu.cn (J.Y.); wangn324@nenu.edu.cn (N.W.); xuezj751@nenu.edu.cn (Z.X.); zhangh306@nenu.edu.cn (H.Z.)

* Correspondence: fanyy033@nenu.edu.cn; Tel.: +86-431-8509-8908

+ These authors contributed equally to this work.

Academic Editors: Atanas G. Atanasov, Karel Šmejkal and Elke Heiss

Received: 9 November 2018; Accepted: 19 December 2018; Published: 22 December 2018

\begin{abstract}
Despite the edible fungus Amillariella mellea possessing a variety of biological activities, its effects on diabetes are still unclear. Polysaccharides are the main bioactive ingredients. In order to destroy the cell wall to obtain more polysaccharides, we used $\mathrm{NaOH}$ solution to extract Amillariella mellea fruiting bodies. The alkali extraction (AAMP) was identified as a polysaccharide-enriched fraction. Using type 2 diabetic rats induced by co-treatment of a high fat diet (HFD) and dexamethasone (DEX), we evaluated the hypoglycemic effects of AAMP. The results showed that oral administration of a high dose of AAMP markedly lowered fasting blood glucose, improving glucose intolerance and insulin resistance. AAMP also enhanced the level of LPL and the expressions of two critical lipases ATGL and HSL, leading to a decrease of serum triglyceride. In addition, AAMP specifically suppressed the expression of SREBP-1c, resulting in AAMP observably inhibiting lipid accumulation in the liver. These findings demonstrated that the improvement of AAMP on HFD/DEX-induced insulin resistance was correlated with its regulation of lipid metabolism. Our results indicated that AAMP could be a novel natural drug or health food used for the treatment of diabetes.
\end{abstract}

Keywords: Amillariella mellea; polysaccharide; insulin resistance; lipid metabolism

\section{Introduction}

Insulin resistance is defined as a reduced ability of tissues or cells to respond to normal levels of insulin, resulting in obesity and type 2 diabetes [1,2]. Accumulating studies suggest the relationship between lipid oversupply and insulin resistance [3,4]. Hyperinsulinemia may increase lipid synthesis and supply to muscles and the liver $[5,6]$. The Randle glucose-fatty acid cycle may contribute to the acute manifestation of insulin resistance in hyperlipidemic states, such as obesity [7]. Growth hormone, corticosteroids, and epinephrine are used to accelerate the release of fatty acids in adipose tissue, and through this cycle to inhibit muscle ingestion of glucose, leading to insulin insensitivity. In addition, excess glucocorticoid has been known to cause insulin resistance by inhibiting glucose transport [8].

Mushrooms are a potential source for functional foods due to their bioactive and non-toxic ingredients. Polysaccharides are mainly in the cell walls and the fruiting bodies of fungi [9]. Amillariella mellea is a popular edible fungus with wide distribution in the northeastern region of China. Previous studies have shown that the polysaccharides from $A$. mellea have potential biological activities, with a focus on anti-inflammation [10], anti-oxidation [11], and immunomodulation [12]. It has also been 
reported that polysaccharide from A. mellea promoted glucose-induced insulin secretion by scavenging the free radicals in alloxan-treated pancreatic cells [13]. However, it is not clear whether polysaccharide from A. mellea have a hypoglycemic effect in vivo.

We recently isolated a polysaccharide-enriched fraction (AAMP) from Amillariella mellea fruiting bodies. Here we evaluate the hypoglycemic activity of AAMP in a high-fat diet and glucocorticoid-induced diabetic rat and identify its mechanisms.

\section{Results}

\subsection{Preparation of the Polysaccharide-Enriched Fraction from Amillariella Mellea Fruiting Body}

The fruiting bodies of Amillariella mellea were defatted with $95 \%$ ethanol and extracted with hot water. The residues were further extracted with $0.5 \mathrm{M} \mathrm{NaOH}$ solution, precipitated with $75 \%$ ethanol, dialyzed with distilled water, and lyophilized; the extraction was named AAMP. It contained $68.4 \%$ total carbohydrates and $14.3 \%$ protein. The monosaccharide composition analysis showed AAMP was composed of glucose (Glc, 58.6\%), galactose (Gal, 19.8\%), mannose (Man, 18.1\%), glucuronic acid (GlcA, 3.3\%), and fucose (Fuc, 1.5\%). The molecular weight distribution of AAMP was determined by HPGPC and two peaks were detected at different retention times. As shown in Figure 1, AAMP was composed of two fractions with molecular weights of $321 \mathrm{kDa}$ and $23.3 \mathrm{kDa}$, respectively, and the latter was the major fraction.

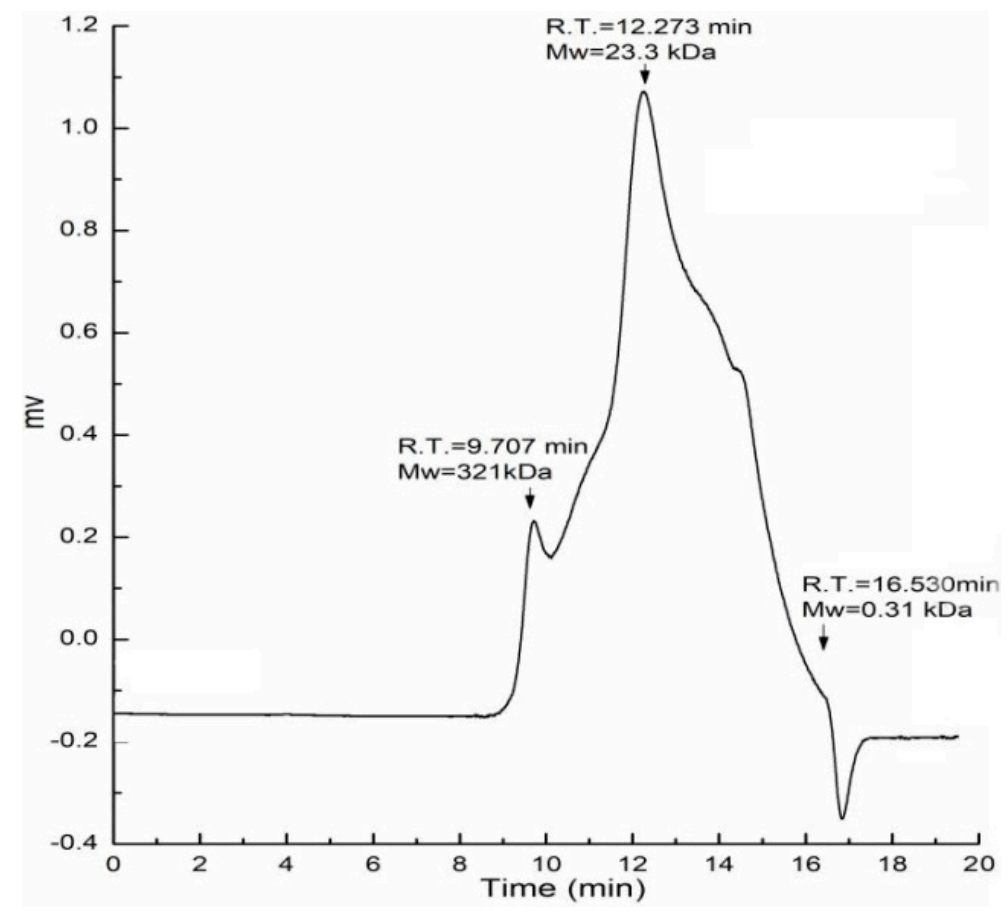

Figure 1. Molecular weight distribution of AAMP.

\subsection{Fourier Transform Infrared Spectroscopy (FT-IR) Analysis}

The FT-IR spectrum of AAMP exhibited typical carbohydrate patterns (Figure 2). The intense and broad peak around $3389 \mathrm{~cm}^{-1}$ indicated the presence of $\mathrm{O}-\mathrm{H}$ stretching in hydrogen bonds [14]. The weak band near $2922 \mathrm{~cm}^{-1}$ was attributed to the $\mathrm{C}-\mathrm{H}$ stretching and bending vibrations. The carbonyl groups $(\mathrm{C}=\mathrm{O})$ were also observed with characteristic absorptions at the asymmetrical stretching band at $1636 \mathrm{~cm}^{-1}$ and symmetric stretching band around $1400 \mathrm{~cm}^{-1}$ [15]. The peak at $1076 \mathrm{~cm}^{-1}$ was assigned to the $\mathrm{O}-\mathrm{H}$ variable angle vibration, and the characteristic band near $1043 \mathrm{~cm}^{-1}$ suggested the presence of pyranose rings. Moreover, the weak peak at $915 \mathrm{~cm}^{-1}$ indicated the presence of $\beta$-linked-D-glucopyranose residues [16]. 


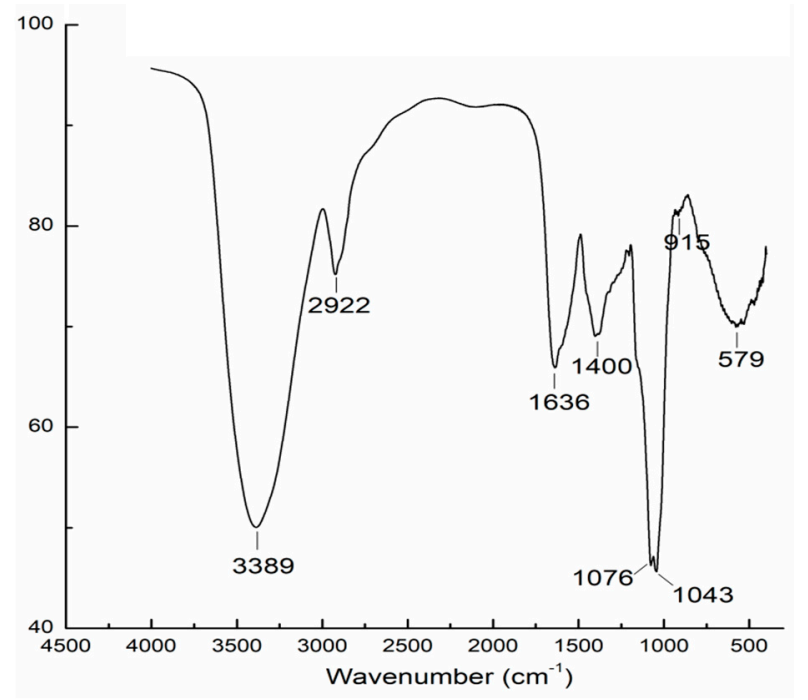

Figure 2. FT-IR spectrum of AAMP.

\subsection{AAMP Reduced Fasting Blood Glucose in HFD/DEX-Treated Rat}

The hypoglycemic effect of AAMP was examined by high-fat diet and dexamethasone co-treated (HFD/DEX) rats. Compared with the body weight of normal rats, HFD/DEX treatment slowed the body weight increase. AAMP did not alter the body weight change of HFD/DEX-treated rats (Figure 3A). As shown in Figure 3B, the fasting blood glucose (FBG) of rats treated with HFD/DEX exerted higher than that of normal rats, suggesting that HFD/DEX might cause insulin resistance. In normal rats, a high dose of AAMP did not affect FBG, but in HFD/DEX-treated rats, AAMP slightly reduced FBG at a low dose $(50 \mathrm{mg} / \mathrm{kg} /$ day $)$, and significantly reduced FBG at a high dose $(200 \mathrm{mg} / \mathrm{kg} /$ day). The results indicated that AAMP specifically reduced blood glucose in insulin-resistant rats.

A

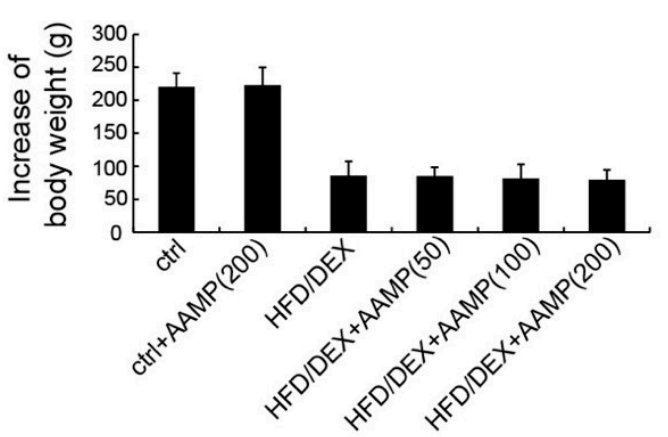

B

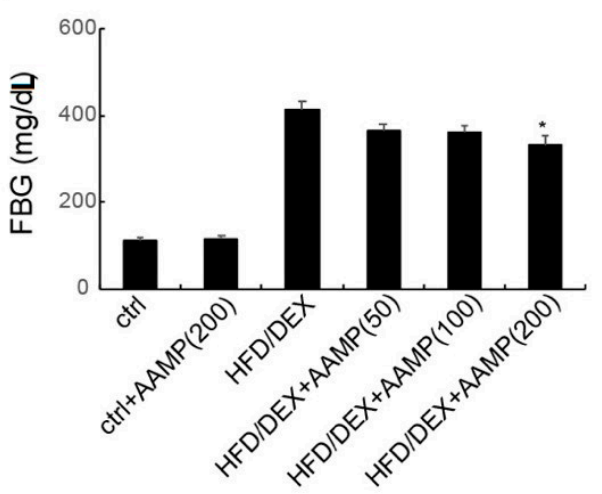

Figure 3. AAMP treatment lowered blood glucose of the diabetic rats. The rats were administered with different doses of AAMP for 35 days. (A) Increase of body weight. (B) Fasting blood glucose. Results represent the mean \pm S.E.M. ( $n=15$ rats in each group). ${ }^{*} p<0.05$ compared to HFD/DEX rats.

\subsection{AAMP Improved Glucose Intolerance in HFD/DEX-Treated Rat}

During glucose tolerance test (GTT), the blood glucose levels of rat treated with HFD/DEX were higher at all considered times compared to normal rats (Figure 4), indicating that HFD/DEX generated glucose intolerance. In HFD/DEX-treated rats, AAMP caused the blood glucose to rise and then fall back quickly to the initial level at a high dose (Figure 4A), and reduced the AUC (Figure 4B), indicating that it obviously improves glucose intolerance. 
A

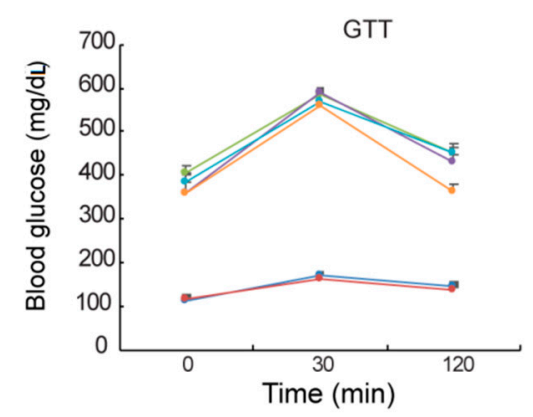

B

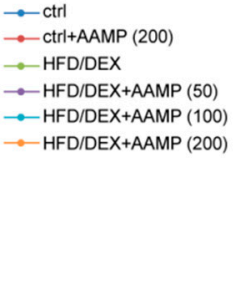

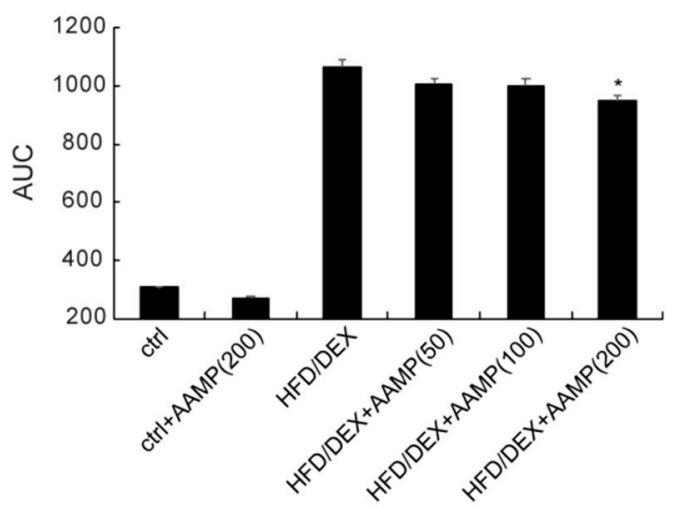

Figure 4. AAMP treatment ameliorated glucose intolerance of the diabetic rats. At the last day of the animal experiment, blood glucose changes were observed at 0, 30, and $120 \mathrm{~min}$ after intragastric administration of glucose in rats. (A) GTT. (B) The calculation of area under the curve (AUC) from GTT. Results represent the mean \pm S.E.M. ( $n=15$ rats in each group). ${ }^{*} p<0.05$ compared to HFD/DEX rats.

\subsection{AAMP Ameliorated Insulin Resistance in HFD/DEX-Treated Rat}

The serum insulin of rat after HFD/DEX treatment was distinctly higher than that of normal rat (Figure 5A), and the HOMA-IR (homeostasis model assessment index for insulin resistance) of the former was also higher than the latter (Figure 5B), suggesting that HFD/DEX caused severe insulin resistance. After administration of AAMP, the serum insulin decreased (Figure 5A) and the HOMA-IR declined (Figure 5B) compared to HFD/DEX-treated rats. These results demonstrated that AAMP improved insulin resistance.

A

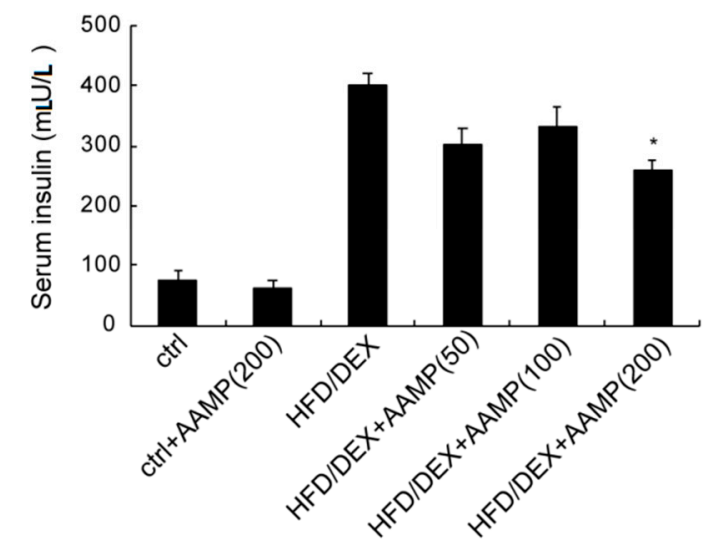

B

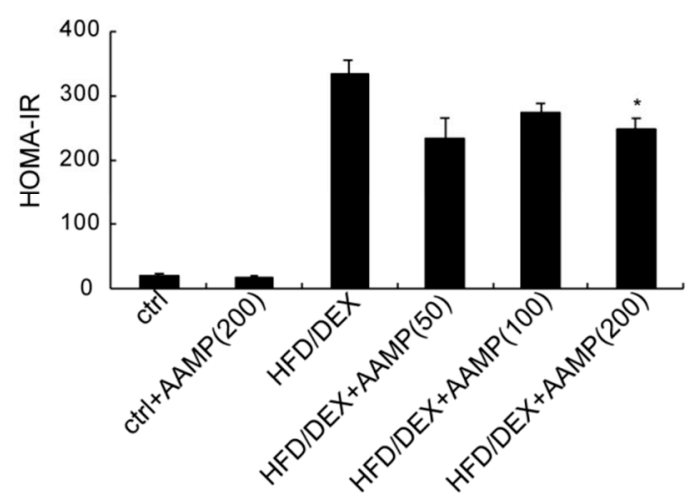

Figure 5. AAMP treatment enhanced insulin sensitivity of the diabetic rats. (A) The content of serum insulin. (B) The calculation of HOMA-IR from FBG and serum insulin. Results represent the mean \pm S.E.M. ( $n=15$ rats in each group). ${ }^{*} p<0.05$ compared to HFD/DEX rats.

\subsection{AAMP Lowered Serum Triglyceride and Free Fatty Acids in HFD/DEX-Treated Rat}

Abnormal lipid metabolism usually causes insulin resistance, so we examined whether AAMP affected lipid metabolism. The serum triglyceride (TG), total cholesterol (TC), and free fatty acids (FFA) of rats after HFD/DEX treatment were increased than those of normal rats (Figure 6A-C), indicating that HFD/DEX affected lipid metabolism. After AAMP administration, the serum TG and FFA significantly decreased, but not TC, compared to HFD/DEX-treated rats (Figure 6A-C). 
Lipoprotein lipase (LPL) is important in lipid metabolism, so we measured LPL contents. The effect of HFD/DEX on LPL was not significant compared to the control diet, however, AAMP could enhance the level of LPL (Figure 6D), indicating that the significant increase in the LPL level could partly be a reason for the decreased serum TG level, and that AAMP probably regulated lipid metabolism in other manners.

A

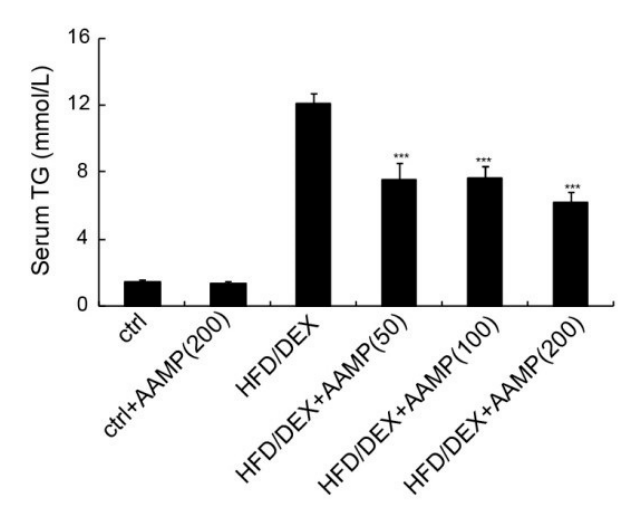

C

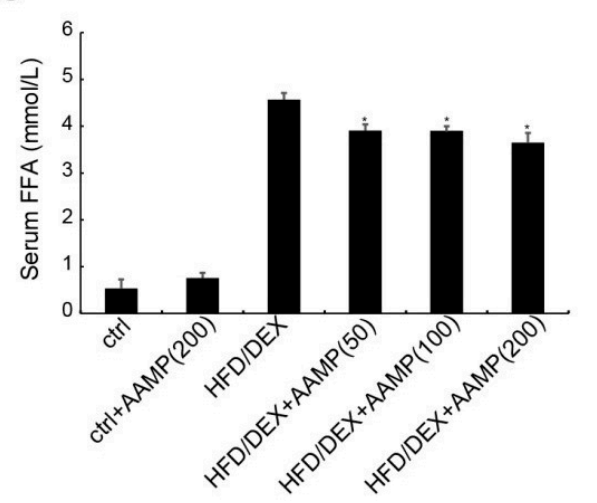

B

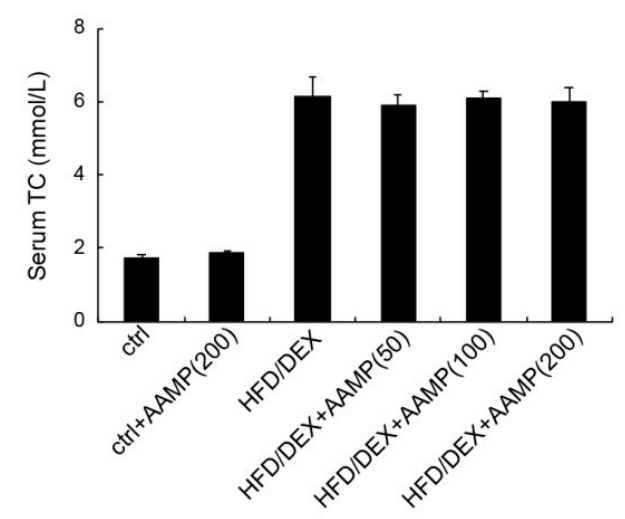

D

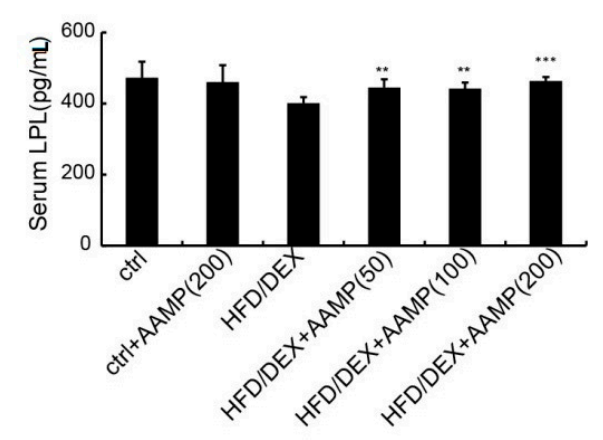

Figure 6. Metabolic profiles of diabetic rats treated with AAMP. (A) Triglyceride (TG). (B) Total cholesterol (TC). (C) Free fatty acid (FFA). (D) Lipoprotein lipase (LPL). Results represent the mean \pm S.E.M. ( $n=15$ rats in each group). ${ }^{*} 0.01<p<0.05 ;{ }^{* *} 0.001<p<0.01 ;{ }^{* * *} p<0.001$ compared to HFD/DEX rats.

\subsection{AAMP Enhanced Lipolysis and Suppressed Lipogenesis}

Since AAMP reduced the contents of serum TG and FFA, we explored the mechanisms of AAMP to regulate lipid metabolism. Adipose triglyceride lipase (ATGL) and hormone-sensitive lipase (HSL) are the two important lipases. AAMP increased the expressions of these two lipases compared to HFD/DEX-treated rats (Figure 7A), indicating that AAMP enhanced lipolysis to decrease TG content. Compared to the normal rat, the muscle cells of the rat became smaller and the cell boundary was blurred after HFD/DEX treatment, and AAMP did not improve rat muscle damage caused by HFD/DEX (Figure 7B). However, AAMP significantly inhibited lipid droplet accumulation in liver (Figure 7B). In addition, the effects of AAMP on lipogenesis transcription factor expressions in liver were also examined. AAMP significantly reduced the expression of SREBP-1c, but no effect on the expression of PPAR $\gamma$ (Figure 7C), suggesting that AAMP inhibited lipogenesis by regulation of transcription factor SREBP-1c. 


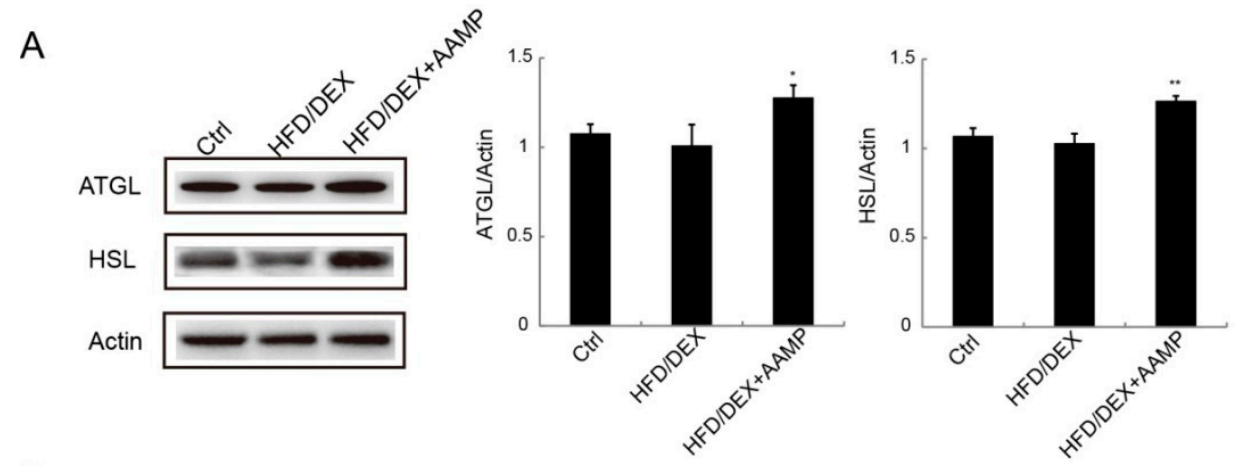

B

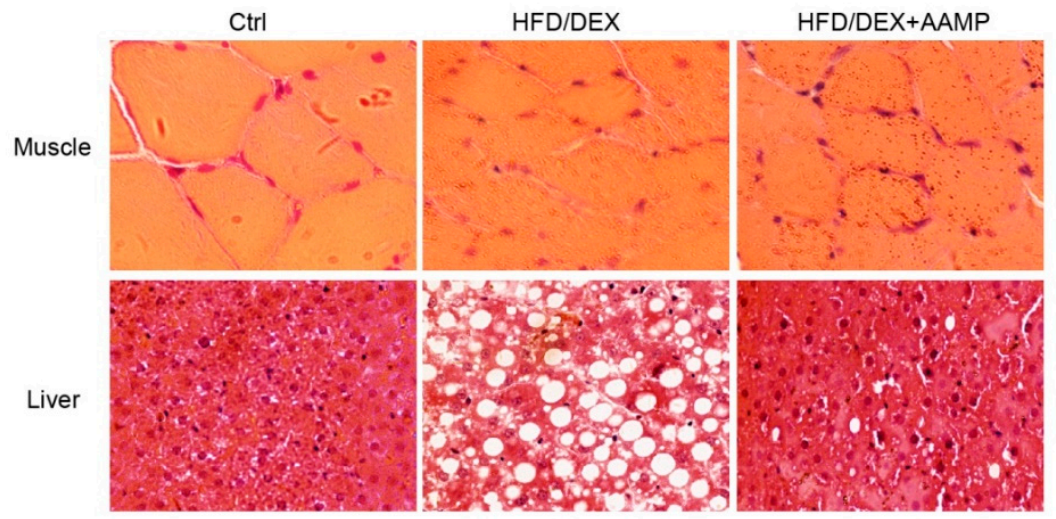

C
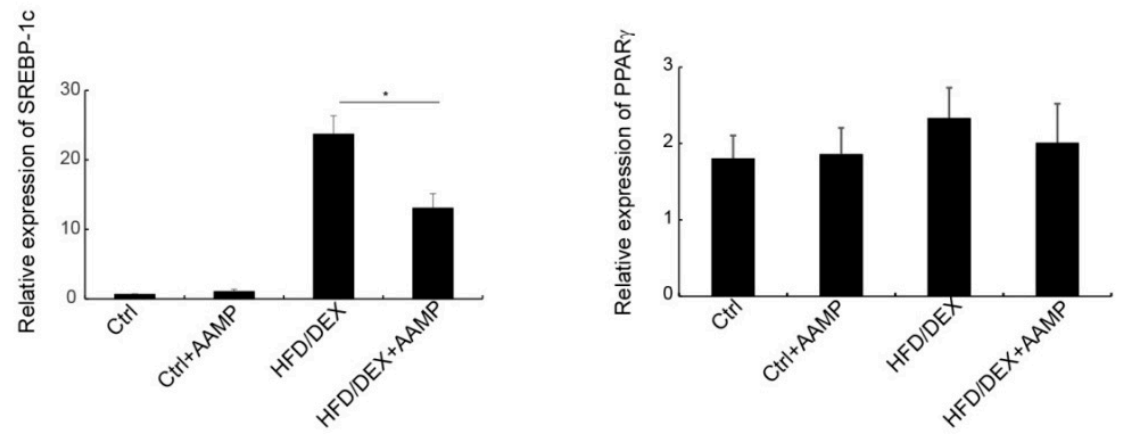

Figure 7. Effects of AAMP on lipid metabolism in diabetic rats. (A) Expressions of lipases in adipose tissue (left) and quantitative analysis (right). (B) The lipid droplets in liver and muscle of the rats. (C) The lipogenesis-related gene expressions in liver. SREBP-1c, sterol regulatory element binding protein 1c; PPAR $\gamma$ peroxisome proliferator-activated receptor $\gamma$ Results represent the mean \pm S.E.M. ( $n=15$ rats in each group). ${ }^{*} 0.01<p<0.05 ;{ }^{* *} 0.001<p<0.01$ compared to HFD/DEX rats.

\section{Discussion}

Long-term feeding with a HFD may increase fat accumulation and high blood glucose in rats [17]. Dexamethasone is a glucocorticoid frequently used to induce insulin resistance by enhancing hepatic glucose output and suppressing the peripheral glucose uptake [18]. Herein, the rats co-treated with HFD and DEX exhibited a loss of body weight, and an increase of fasting blood glucose, serum insulin levels, and triglycerides, which are typical features of type 2 diabetes [19]. The indicators of HFD/DEX-treated rats observed in our experiments are consistent with the literature, indicating that we successfully established a rat model of type 2 diabetes. The polysaccharide-enriched fraction AAMP from Amillariella mellea fruiting body exerts an oral hypoglycemic effect at high dose by lowering fasting blood glucose, improving glucose-intolerance, and reducing serum insulin, TG, and FFA levels.

Dexamethasone treatment is associated with an increase of triglyceride production rates and hypertriglyceridemia [20]. The triglycerides are hydrolyzed step by step to produce free fatty acid and glycerol. We found that AAMP increases the level of LPL and the expressions of two main lipases, 
ATGL and HSL, in HFD/DEX-treated rat, resulting in a decrease of triglycerides. Fatty acids through the bloodstream are absorbed by liver or muscle to participate in lipid metabolism. AAMP specifically reduces the number of lipid droplets in liver, but has no effect on fat deposition in muscle. It is speculated that AAMP also affects lipogenesis in the liver. Lipogenesis is controlled by multiple transcriptional regulators, such as SREBP and PPAR. SREBP-1c activates genes involved in fatty acid and TG synthesis. ER stress stimulates the activation of SREBP-1c to increase in lipogenesis [21]. PPAR $\gamma$ controls fatty acid uptake, trafficking, and TG synthesis, ablation of hepatic PPAR $\gamma$ alleviates liver steatosis in obese mice [22,23]. AAMP specifically suppressed the expression of SREBP-1c, but not PPAR $\gamma$, indicating that AAMP probably regulated fatty acid and TG synthesis. Therefore, the hypoglycemic effect of AAMP is likely related to increased lipolysis and decreased lipogenesis.

AAMP could regulate lipid metabolism to lower blood glucose in hyperglycemic rats and had no toxic side effects on normal rats, suggesting that AAMP has the potential to develop hypoglycemic health foods and drugs.

\section{Materials and Methods}

\subsection{Preparation of the Polysaccharide-Enriched Fraction}

Fruiting bodies of Amillariella mellea were collected from Changbai Mountain in Jilin Province, China and identified using rDNA-ITS sequencing analysis. Fruiting bodies were defatted with $95 \%$ ethanol, and the residues were extracted with distilled water three times at $100{ }^{\circ} \mathrm{C}$ for $4 \mathrm{~h}$. After filtration, the residues were dried and extracted using $0.5 \mathrm{M} \mathrm{NaOH} / \mathrm{NaBH}_{4}$ at $80^{\circ} \mathrm{C}$. Extracts were neutralized with glacial acetic acid, and precipitated with $75 \%$ ethanol. The precipitate was collected and lyophilized. The alkali extraction obtained from Amillariella mellea was named AAMP. The total carbohydrate content, protein content, and monosaccharide composition were also detected according to classical methods [24-26]. The molecular weight distribution of AAMP was determined by using gel-permeation chromatography on a Shimadzu HPLC system (Shimadzu, Tokyo, Japan) coupled with a TSK-gel G-3000PWXL column $(7.8 \times 300 \mathrm{~mm}$, TOSOH, Tokyo, Japan) as described by Zhang [27]. The column was pre-calibrated by using standard dextrans (50 kDa, $25 \mathrm{kDa}, 12 \mathrm{kDa}$, $5 \mathrm{kDa}$, and $1 \mathrm{kDa}$ ) with linear regression [27].

\subsection{Fourier Transform Infrared Spectroscopy Analysis}

Structures of polysaccharides, such as glycosidic bonds and functional groups, can be analyzed using the FT-IR spectroscopy. FT-IR spectrum of AAMP was determined on a Spectrum Two spectrometer (PerkinElmer, Waltham, MA, USA) in a range of $4000-400 \mathrm{~cm}^{-1}$. The sample was ground with $\mathrm{KBr}$ powder and then pressed into $1 \mathrm{~mm}$ pellets for FT-IR measurements.

\subsection{Experimental Protocols}

Male Sprague Dawley rats (SYXK 2018-0015) (180-200 g) were obtained from Model Animal Research Center of Nanjing University (Nanjing, China). The animals were housed in a room maintained at $23 \pm 2{ }^{\circ} \mathrm{C}$ with relative air humidity of $45 \%$ to $55 \%$ on a $12 \mathrm{~h}$ light/12 h dark cycle. Before treatment, the fasting blood glucose (FBG) and oral glucose tolerance of the rats were examined as the basic values. The rats were divided into six groups $(n=15)$, including the control group, control plus high dose of AAMP ( $200 \mathrm{mg} / \mathrm{kg} /$ day) group, model group, model plus low dose of AAMP (50 mg/ $\mathrm{kg} /$ day) group, model plus moderate dose of AAMP (100 mg/kg/day) group, and model plus high dose of AAMP $(200 \mathrm{mg} / \mathrm{kg} /$ day $)$ group. The control groups were given normal chow diet ( $10 \% \mathrm{kcal} \%$ fat). The model groups were continuously fed with $60 \% \mathrm{kcal} \%$ fat diet (HFD, Research Diets, D12492) for 35 days, and dexamethasone $(0.8 \mathrm{mg} / \mathrm{kg}$, i.p) was additionally administered on the last 10 days. The rats treated with AAMP were intragastrically administered for 35 days, once daily. 


\subsection{Fasting Blood Glucose Test}

The rats were fasted for $4 \mathrm{~h}$. The blood was collected from tail and measured by the One Touch Ultra Easy Glucometer (Johnson, New Brunswick, NJ, USA).

\subsection{Glucose Tolerance Test (GTT)}

The rats were fasted for $4 \mathrm{~h}$ followed by intragastrically administered with $2.5 \mathrm{~g} / \mathrm{kg}$ body weight of glucose. Blood glucose levels were monitored at 0,30, and $120 \mathrm{~min}$ after administration using blood collected from the tail using the One Touch Ultra Easy Glucometer.

\subsection{Biochemical Indicators Detection}

The serum level of insulin was measured using a commercially available kit (IBL international, Hamburg, Germany). The homeostasis model assessment index for insulin resistance (HOMA-IR) was defined as $($ FBG $\times$ serum insulin)/22.5. The contents of serum triglyceride (TG), total cholesterol (TC), and free fatty acid (FFA) were measured by the kits (Jiancheng, Nanjing, China). The content of serum lipoprotein lipase (LPL) was measured using an ELISA kit (AMEKO, Shanghai, China).

\subsection{H and E Staining}

The liver and muscle were fixed in 4\% paraformaldehyde (Sangon Biotech, A500684-0500, Shanghai, China), and then embedded in paraffin, and sectioned at $5 \mu \mathrm{m}$. $\mathrm{H}$ and E staining was performed following standard protocols. Photos were taken using a light microscope (OLYMPUS BX51, Tokyo, Japan).

\subsection{Western Blots}

The adipose tissues were lysed with lysis buffer containing $50 \mathrm{mM}$ Tris (pH 7.4), $150 \mathrm{mM} \mathrm{NaCl}$, $1 \mathrm{mM}$ EDTA, 1\% Triton X-100, proteinase inhibitor cocktail (Roche Applied Sciences, Penzberg, Upper Bavaria, Germany) and phosphatase inhibitor cocktail (Thermo Scientific, Waltham, MA, USA). Western blots were performed as described in the previous publication [24] using the following primary antibodies: anti-ATGL (Abcam, Cambridge, UK, ab109251, 1:1000), anti-HSL (Sigma, Milpitas, CA, USA, SAB4501763, 1:1000), and anti-actin (Abcam, 1:1000) antibodies.

\subsection{Quantitative Real-Time PCR (RT-qPCR)}

Total RNA of the tissues was extracted using Trizol (Thermo Fisher, Waltham, MA, USA) according to the instructions provided by the manufacturer. cDNA was generated from $1 \mu \mathrm{g}$ of RNA using M-MLV reverse transcriptase (Promega, Fitchburg, WI, USA). qPCR was performed using SYBR Green real-time PCR master mixes (Thermo Fisher, Waltham, MA, USA) and a LightCycler 480 real-time PCR system (Roche Applied Science, Indianapolis, IN, USA). qPCR primer sequences are listed in Table 1.

Table 1. The primers used in our study.

\begin{tabular}{ccc}
\hline Gene Symbol & Forward $\left(\mathbf{5}^{\prime} \mathbf{-} \mathbf{3}^{\prime}\right)$ & Reverse $\left(\mathbf{5}^{\prime} \mathbf{-} \mathbf{3}^{\prime}\right)$ \\
\hline SREBP-1c & GGAGCCATGGATTGCACATT & GCTTCCAGAGAGGAGGCCAG \\
\hline PPAR $\gamma$ & CCCTTTACCACGGTTGATTTCTC & GCAGGCTCTACTTTGATCGCACT \\
\hline GAPDH & ATGATTCTACCCACGGCAAG & CTGGAAGATGGTGATGGGTT \\
\hline
\end{tabular}

\subsection{Statistical Analysis}

The results were expressed as the mean \pm S.E.M. Statistical analysis of the data was performed using Student's t-test and two-way repeated-measures ANOVA with Dunnett's post-hoc test (IBM SPSS Statistics 17.0, Armonk, NY, USA). Differences were considered significant when $p<0.05$. 
Author Contributions: All authors participated in the design of the study. S.Y. and Y.M. prepared the polysaccharide-enriched fraction and were responsible for most animal experiments. J.Y. detected the properties of the fraction. N.W. examined biochemical indicators of the rats. Z.X. and H.Z. performed lipid metabolic assays. Y.F. contributed to data interpretation and the manuscript.

Funding: This work was supported by National Natural Science Foundation of China (No. 31872674), the Scientific and Technologic Foundation of Jilin Province (No. 20180311068YY), Jilin Province Development and Reform Commission (2018C047-2), and the Fundamental Research Funds for the Central Universities (No. 2412017FZ018).

Conflicts of Interest: The authors have declared no conflicts of interest. The founding sponsors had no role in the design of the study; in the collection, analyses, or interpretation of data; in the writing of the manuscript, and in the decision to publish the results.

\section{References}

1. Farese, R.V., Jr.; Zechner, R.; Newgard, C.B.; Walther, T.C. The problem of establishing relationships between hepatic steatosis and hepatic insulin resistance. Cell Metab. 2012, 15, 570-573. [CrossRef] [PubMed]

2. Kahn, S.E.; Hull, R.L.; Utzschneider, K.M. Mechanisms linking obesity to insulin resistance and type 2 diabetes. Nature 2006, 444, 840-846. [CrossRef] [PubMed]

3. Boden, G. Effects of free fatty acids (FFA) on glucose metabolism: Significance for insulin resistance and type 2 diabetes. Exp. Clin. Endocrinol. Diabetes 2003, 111, 121-124. [CrossRef] [PubMed]

4. Guilherme, A.; Virbasius, J.V.; Puri, V.; Czech, M.P. Adipocyte dysfunctions linking obesity to insulin resistance and type 2 diabetes. Nat. Rev. Mol. Cell Bio. 2008, 9, 367-377. [CrossRef]

5. Koves, T.R.; Ussher, J.R.; Noland, R.C.; Slentz, D.; Mosedale, M.; Ilkayeva, O.; Bain, J.; Stevens, R.; Dyck, J.R.B.; Newgard, C.B.; et al. Mitochondrial overload and incomplete fatty acid oxidation contribute to skeletal muscle insulin resistance. Cell Metab. 2008, 7, 45-56. [CrossRef] [PubMed]

6. Tanaka, T.; Yamamoto, J.; Iwasaki, S.; Asaba, H.; Hamura, H.; Ikeda, Y.; Watanabe, M.; Magoori, K.; Ioka, R.X.; Tachibana, K.; et al. Activation of peroxisome proliferator-activated receptor $\delta$ induces fatty acid $\beta$-oxidation in skeletal muscle and attenuates metabolic syndrome. Proc. Natl. Acad. Sci. USA 2003, 100, 15924-15929. [CrossRef] [PubMed]

7. Perry, R.J.; Wang, Y.; Cline, G.W.; Rabin-Court, A.; Song, J.D.; Dufour, S.; Zhang, X.M.; Petersen, K.F.; Shulman, G.I. Leptin Mediates a Glucose-Fatty Acid Cycle to Maintain Glucose Homeostasis in Starvation. Cell 2018, 172, 234-248. [CrossRef] [PubMed]

8. Priyadarshini, E.; Anuradha, C.V. Glucocorticoid Antagonism Reduces Insulin Resistance and Associated Lipid Abnormalities in High-Fructose-Fed Mice. Can. J. Diabetes 2017, 41, 41-51. [CrossRef] [PubMed]

9. Ooi, V.E.C.; Liu, F. Immunomodulation and anti-cancer activity of polysaccharide-protein complexes. Curr. Med. Chem. 2000, 7, 715-729. [CrossRef]

10. Lee, S.; Lee, D.; Park, J.Y.; Seok, S.; Jang, T.S.; Park, H.B.; Shim, S.H.; Kang, K.S.; Kim, K.H. Antigastritis effects of Armillariella tabescens (Scop.) Sing. and the identification of its anti-inflammatory metabolites. J. Pharm. Pharmacol. 2018, 70, 404-412. [CrossRef] [PubMed]

11. Ng, L.T.; Wu, S.J.; Tsai, J.Y.; Lai, M.N. Antioxidant activities of cultured Armillariella mellea. Prikl. Biokhim. Mikrobiol. 2007, 43, 495-500. [CrossRef] [PubMed]

12. Chen, F.; Huang, G.L. Preparation and immunological activity of polysaccharides and their derivatives. Int. J. Biol. Macromol. 2018, 112, 211-216. [CrossRef] [PubMed]

13. Lu, J.; Cao, Y.P.; Yu, M.; Shen, Y.S. Polysaccharide from the rhizomorph of Armillaria mella (AMP-1) protects INS-1 cells from alloxan injury. Chin. Pharmacol. Bull. 2008, 24, 1160-1165.

14. Kozarski, M.; Klaus, A.; Niksic, M.; Vrvic, M.M.; Todorovic, N.; Jakovljevic, D.; Griensven, L.J. Antioxidative activities and chemical characterization of polysaccharide extracts from the widely used mushrooms Ganoderma applanatum, Ganoderma lucidum, Lentinus edodes and Trametes versicolor. J. Food Compos. Anal. 2012, 26, 144-153. [CrossRef]

15. Casu, B.; Scovenna, G.; Cifonelli, A.J.; Perlin, A.S. Infra-red spectra of glycosaminoglycans in deuterium oxide and deuterium chloride solution: Quantitative evaluation of uronic acid and acetamidodeoxyhexose moieties. Carbohydr. Res. 1978, 63, 13-27. [CrossRef]

16. Barker, S.A.; Bourne, E.J.; Stacey, M.; Whiffen, D.H. Infra-red spectra of carbohydrates. Part I. Some derivatives of D-glucopyranose. J. Chem. Soc. 1954, 1, 171-176. [CrossRef] 
17. Zabielski, P.; Hady, H.R.; Chacinska, M.; Roszczyc, K.; Gorski, J.; Blachnio-Zabielska, A.U. The effect of high fat diet and metformin treatment on liver lipids accumulation and their impact on insulin action. Sci. Rep. 2018, 8, 7249. [CrossRef] [PubMed]

18. Ogawa, A.; Johnson, J.H.; Ohneda, M.; McAllister, C.T.; Inman, L.; Alam, T.; Unger, R.H. Roles of insulin resistance and beta-cell dysfunction in dexamethasone-induced diabetes. J. Clin. Invest. 1992, 90, 497-504. [CrossRef] [PubMed]

19. Abildgaard, A.; Lund, S.; Hougaard, K.S. Chronic high-fat diet increases acute neuroendocrine stress response independently of prenatal dexamethasone treatment in male rats. Acta Neuropsychiatr. 2014, 26, 8-18. [CrossRef] [PubMed]

20. Kumar, V.S.; Inamdar, M.N.; Viswanatha, G.L. Protective effect of lemongrass oil against dexamethasone induced hyperlipidemia in rats: Possible role of decreased lecithin cholesterol acetyl transferase activity. Asian Pac. J. Trop. Med. 2011, 4, 658-660. [CrossRef]

21. Kim, J.Y.; Garcia-Carbonell, R.; Yamachika, S.; Zhao, P.; Dhar, D.; Loomba, R.; Kaufman, R.J.; Saltiel, A.R.; Karin, M. ER Stress Drives Lipogenesis and Steatohepatitis via Caspase-2 Activation of S1P. Cell 2018, 175, 133-145. [CrossRef] [PubMed]

22. Kubota, N.; Terauchi, Y.; Miki, H.; Tamemoto, H.; Yaauchi, T.; Komeda, K.; Satoh, S.; Nakano, R.; Ishii, C.; Sugiyama, T.; et al. PPAR $\gamma$ mediates high-fat diet-induced adipocyte hypertrophy and insulin resistance. Mol. Cell 1999, 4, 597-609. [CrossRef]

23. Souza-Mello, V. Peroxisome proliferator-activated receptors as targets to treat non-alcoholic fatty liver disease. World J. hepatol. 2015, 7, 1012-1019. [CrossRef] [PubMed]

24. Gong, H.Q.; Li, X.Z.; Yang, S.W.; Yang, L.L.; Fan, Y.Y.; Zhou, Y.F. Pectic Bee Pollen Polysaccharide from Rosa rugosa Alleviates Diet-Induced Hepatic Steatosis and Insulin Resistance via Induction of AMPK/mTOR-Mediated Autophagy. Molecules 2017, 22, 699. [CrossRef]

25. Sedmak, J.J.; Grossberg, S.E. A rapid, sensitive, and versatile assay for protein using Coomassie brilliant blue G250. Anal. Biochem. 1997, 79, 544-552. [CrossRef]

26. Blumenkrantz, N.; Asboe-Hansen, G. New method for quantitative determination of uronic acids. Anal. Biochem. 1973, 54, 484-489. [CrossRef]

27. Zhang, X.; Yu, L.; Bi, H.T.; Li, X.H.; Ni, W.H.; Han, H.; Li, N.; Wang, B.Q.; Zhou, Y.F.; Tai, G.H. Total fractionation and characterization of the water-soluble polysaccharides isolated from Panax ginseng $C A$ Meyer. Carbohydr. Polym. 2009, 77, 544-552. [CrossRef]

Sample Availability: Samples of the compounds are available from the authors.

(C) 2018 by the authors. Licensee MDPI, Basel, Switzerland. This article is an open access article distributed under the terms and conditions of the Creative Commons Attribution (CC BY) license (http:/ / creativecommons.org/licenses/by/4.0/). 